\title{
DIE ERSTELLUNG EINES BILDERWÖRTERBUCHES ZUM THEMA NOMEN IN DER SCHULE
}

\author{
Gloria Agustina Saragih \\ Ahmad Sahat Perdamean \\ Herlina Jasa Putri Harahap
}

Das Ziel dieser Untersuchung ist es, um ein Bilderwörterbuch zum Thema Nomen in der Schule $\mathrm{zu}$ erstellen. DieErstellungsuntersuchung wirdin dieser Untersuchung angewendet. Der Prozess der Erstellung eines Bilderwörterbuches zum Thema Nomen in der Schule mithilfe der Applikaton Videoshow besteht aus der Erklärung der Phasen von Plomps Theorie. Darunter sind: (1) die Beobachtungsphase, (2) die Designsphase, (3) die Realisierungs-oder Konstruktionsphase und (4) die Revisionsphase.Die Daten und Datenquelle dieser Untersuchung sinddie Wörter über Nomens in der Schule und stammen aus dem Buch, Regenbogen, das Deutschwörterbuch, Jung und anderen Bücher stammen.Das Ergebnis derErstellung eines Bilderwörterbuches zum Thema Nomen in der Schuleist der Wortschatz des Nomens in der Schule mit den Bilder und einem Beispielsatz.Die Bewertungder Expertin über die Qualität des Ergebnisses derErstellung eines Bilderwörterbuches zum Thema Nomen in der Schuleist sehr gut.

Schlüsswörter: Die Erstellung, Bilderwörterbuch, Nomen.

\section{EINLEITUNG}

\section{A. Hintergrund}

Bei einer Fremdsprache gibt es die vier Kompetenzen, Hören, Lesen, Schreiben und Sprechen, die man beherrschen muss. Wander (in Rahayu, 2013:2) sagt, dass diese Kompetenzen nicht vomWörtschatz getrennt werden können. Sie hängen miteinander zusammen. Tschimer (2010: 236) stellt fest, dass der Wortschatz die Gesamtmenge aller Wörter einer Sprache oder einer Person zu einem bestimmten Zeitpunkt ist. Also die Beherrschung des guten Wortschatzes wird im Deutschlernen benutzt. Das bedeutet, ohne das Wortschatz kann man die vier Kompetenzen nicht erreichen.

Hardono(in Choirul, 2014:10) sagt aus, dass man eine Fremdsprache ohne die Beherrschung des Vokabulars nicht sprechen kann. Es gibt viele Wege, um den Wortschatz beim Deutschlernen zu vergrößen. Einer davon ist die Verwendung des Wörterbuches. Das Wörterbuch, das zumeinst benutzt wird, ist 
von Adolf Heuken (schwarz und weiß). Aber viele Schüler verwenden das Wörterbuch nicht, da es Wörterbuch ist uninteressant und die Schüler zu faul sind das Wörterbuch zu lesen, Deshalb benötigen die Schüler andere Hilfsmittel, zum Beispiel ein Bilderwörterbuch. Daswird selten noch im Deustchunterricht benutzt.

Das Bilderwörterbuch kann die Motivation und auf die Aufmerksamkeit der Schüler im Lernprozess steigern, denn es enhält interessante Bilder. In den”Blitung Tribun News"aus dem Jahr 2017 wird beschreiben, dass Android nutzen können, um eine Fremdsprache zu lernen, das es mittlerweile viele Apps und Informationen gibt, die man herunterladen kann und die den Lernprozess unterstützen können.

Basierend auf dieser Ausgangslage hat die Verfasserin die Idee, ein Bilderwörterbuch zum Thema Nomen in der Schule mithilfe der Applikation Videoshow $\mathrm{zu}$ erstellen, um den deutschen Wortschatz der Schüler zu vergrößen.Die Verfasserin hat das Videoshow ausgewählt, weil es eine App auf des Basis von android ist und somit für die Schüler einfach zugänglich und leicht verständlich ist, da sie auf dem Handy abgespielt werden kann und somit den Lernprozess angenehmer macht. Das Thema Nomen in der Schüle wird ausgewählt, weil das Thema interresant istund an einigen Schülern und Universitäten gelernt wird.

\section{THEORETISCHE GRUNDLAGE}

\section{Der Bergriff der Entwicklungsuntersuchung}

Borg und Gall (1998:28) stellen fest, dass die Forschung oder die Entwicklung ein Prozess oder eine Methode ist, die verwendet wird, umProdukte $\mathrm{zu}$ validieren und entwickeln.Sukmadinata (2009:164) behauptet, dass es der Prozess oder die Schritte sind, die Unternommen werden, um ein neues Produkt zu erstellen oder ein bestehendes Produkt zu vervollkommen.

Es kann also zusammengefasst werden, dass die Forschung und Die Entwicklung sind ein Prozess sind, um die Produkte zu validieren und entwickeln.

Der Bergriff des Bilderwörterbuches 
Das Bilderwörterbuch ist ein Nachschlagewerk, das Wörter enthält, die mithilfe von den Bildern und Erklärungen erklärt werden sollen. Anneahira (in Dewi, 2014:27) stellt fest, dass das Bildwörterbuch das Wörterbuch ist, das interessante Bilder enthält und die aufmerksamkeit der Menschen erhöhen, besonders für Kinder in der Lernphase.Im Duden (1983:201) wird ein Bilderwörterbuch als ein Wörterbuch beschrieben, wo die Bedeutung der Wörter durch Bilder erklärt wird.

Es kann also zusammengefasst werden, dass Bilderwörterbuch ist ein Buch, das es der Wörter mit interessanten Bilder hat.

\section{Der Bergriff des Videoshows}

Videoshow ist im Android-app. Die Android-App wird von vielen Menschen verwendet. Videoshow ist eine Applikation am Videos aufnehmen und bearbeiten zu können. Es ist im Playstore die am zweitmeisten heruntesgeladene App, da ihre Verwendung sehr einfach ist (in Musdeoranje:2017).

\section{Der Bergriff des Nomens}

Das Nomen ist eine Wortart, die Menschen, eine Sache oder ein Tier bezeichnet und im Deutschen am Anfang mit einem großen Buchstaben geschrieben wird. Es wird immer einem Artikel verbunden und kann am sowohl im Singular und als auch Plural stehen. Meibauer stellt fest (2007:43), dass jedes Nomen hinsichtlich der drei Merkmalklassen Kasus, Numerus ung Genus spezifiziert. Röhe und Blick (in Fraudina, 2015:6) stellt fest, dass Nomen Namen für Sachen und gedachte Bergriffe sind. Nomen sind Worter, dieMenschen, Tiere, Objekt und Konzept oder das Verständnis benutzt werden (Vicosta, 2011:95). Hüber (1970:43)stellt fest, dass Nomen Lebewesen, Dinge und Begriffe bezeichnen.Es kann also zusammengefasst werden, dass das Nomen : Ein Wort, das die Menschen, die Sache, das Tier, Konzept und Veständnis bezeichnet.

\section{Die konzeptuellen Grundlagen}

Das Nomen ist Ein Wort, das die Menschen, die Sache, das Tier oder Konzept beschreibt. Es ist wichtig für die Schüler zu lernen, besonders für Nomen in der Schule, aber viele Schüler kennen noch nicht viele Nomen. Das 
Untersuchungziel ist es, den Wörtschatz und das Vokabular des Schülers vergrößern. Deshalb erstellt die Verfasserin ein Bilderwörterbuch mit dem Thema „Nomen in der Schule”, denn es istinteressant zu erstellenund es wird in einigen Schüler und Universitäten gelernt. Normallerweise lernen die Schüler Nomen von der 1. Zur 3. Klasse.

Diese Forschung benutzt eine Android-Applikation, um das Bilderwörterbuches zu erstellen. Diese Applikation heißtVideoshow. Videoshow ist eine Bearbeitungappvon Videos. Diese App wird in der Erstellung des Bilderwörterbuch verwendet, weil dieses Video sehr praktisch ist, nutzt effektive beim Lernen und die Verwendung des Videos ist einfach auch

Dieses Untersuchung benutzt das Modell von Plomp, das ausvier Schritten besteht: die Phase der Untersuchung, 1) der Phase der Ermittlung (2) der Phase des Designs (3) der Phase der Realisierung oder der Kontrukstion (4) die Phasen der Prüfung, Bewertung und Revision. Die Phase der Theorie von Plomp sind sehr übersichtlich und sind schnellerund einfacht anzuwenden.

\section{UNTERSUCHUNGMETHODE}

Diese Untersuchung ist eine Entwicklungsuntersuchung. Es wird ein Bilderwörterbuch zum Thema Nomens in derSchule mithilfeVideoshow erstellt. Diese Untersuchung benutzt die deskriptive qualitative Methode.

\section{Die Daten Und Datenquelle}

Die Quelle von dieser Untersuchung sind die Wörter über Nomens in der Schlue und stammen aus dem Buch, Regenbogen, das Deutschwörterbuch, Jung.Diese Untersuchung wird in der Bibliothek der Fakultät für Sprache und Kunst durchgeführt. Diese Untersuchung benutzt die Theorie von Plomp.

\section{ERGEBNIS DER UNTERSUCHUNG Daten Beschreibung}

In diesem Kapitel werden der Prozess und die Ergebnisse der Erstellung eines Bilderwörterbuches zum Thema" Nomen in der Schule" für Anfänger erklärt. Die Schritte und die Ergebnisse der Untersuchung werden in diesem Kapitel erklärt. 


\section{Der Prozess der Erstellung}

\section{Die Ermittlunsphase}

In dieser Phase werden einige Bücher gelesen, um Nomen in der Schule zu sammeln. Nachdem die Bücher gelesen wurde, werden die Nomen im Buch markiert und gesammelt. Andere Nomen können aus anderen Quellen gesammelt. Anschließen wird jedes Nomen vom Indonesischen ins Deutscheübersetzt. Zuletz werden passende Bilder zu diesen Nomen gesucht.Nachdem die Daten reduziert wurden, werden130 Nomen gefunden und Alphabetisch.

\section{Designphase}

In dieser zweiten Phase wird das Design durchgeführt, das auf dem Ergebnis der ersten Phase basiert. Das Nomen, das geordnet wird, 9 Videos geteilt.In dieser Phase werden Bilder geordnet. Zuerst wird die Startseite des Wortschatzes bearbeitet. Das Designkonzept beginnt mit der Startseite, dann kommt Nomen in der Schule. Es gibt Singular und Plural, und endlich kommt der Beispielsatz des Nomens in der Schule.

Im Video gibt es unterschiedliche Effect und unterschiedliche Musik, aber im Video hat gleiches Schreiben und gleiche Farbe.In dieser Phase wird das Konzept der Erstellung eines Bilderwörterbuches zum Thema Nomen in der Schule mithilfe der Applikation Videoshow.

Unten sind die Schritte der Erstellung eines Bilderwörterbuches des Nomens in der Schule.

1) Zuerst wird Videoshowgeöffnet. Danach klickt man „Edit Video”, wie in der folgenden Abbildung.

2) Dann klickt man einen Ordnen. Um die Bilder und die Startseite zu wählen.

3) Nachdem die Bilder gewählt wird, klickt man ,Mulai”.

4) Dann muss man,,Tema” oder einen Effekt für ein Video wählen. z.B das Free love Tema. Man kann es klicken.

5) Danach wird die Zeit/ Dauer des Bildes bestimmt.

6) Um die Musik zu wählen, wird die Musik geklicken.. z.B „P Positive

Summer Rock. 
7) Dann klickt man, „Sunting”. Es gibt viele Fitur. z.B „Edit klip, Teks Bawah und Efek, Sulih Suara”. Um die Textart zu wählen, wird „Text Bawah” geklickt und die Nomen geschrieben, danach klickt man „Setelan font” und man klickt Das Symbol ,aa” wie in der folgenden Abbildung. Es gibt viele Textart, dann man kann klickt irgendeine Textart. z.B „Impact” wählen.

8) Um die Textfarbe zu wählen, wird das Symbol der Farbe geklickt. Wie in der folgenden Abbildung. Dann klickt man irgendeine Farbe, z.B die blaue Farbe.

9) Um den Stil des Texts zu wählen, wird das Symbol der Text geklickt. Wie in der folgenden Abbildung. Dann man klickt irgendeinen den Stil des Texts, z.B Cat.

10) Das erste Bilde ist das Wort des nomens und das zweite Bild ist Satz.

11) Danach klickt man „Efek” für einen Bild. Wie in der folgenden Abbildung.

12) Dann klickt man „Sulih suara”, um die Stimme für die Nomen und die Sätze zum Aufzunehmen.

13) Wenn alles bearbeiten ist, kann man den Pfeilklicken zum Video speichern.

14) Dann wird das Video gespeichert und man kann es im Ordnen „Karya saya”anschauen oder man kann „Galeri” öffnen zu anzuschauen.

\section{Realisierungs- oder Konttruktionspahase}

Das Konzept, das in der zweiten Phase geplant wurde, wird in dieser Phase verwirklicht. Die Bilder und Wörtschätze zum Thema Nomen in der Schule werden realisiert.

\section{Revisionsphase}

Das ist der letzteSchritt, um eineinteressante CD eines Bilderwörterbuches zum Thema Nomen in der Schule zuerstellen. In dieser Phase wurdeschon das CD eines Bilderwörterbuches zum Thema "Nomen in der Schulemithilfe der ApplikationVideoshow erstellt. DieserWortschätze wird von Experten geprüft. Die Experten sind Dozenten, deutsche Muttersprachler und Designexperten.

Die Experten haben einen pädagogischen Hintergrund.Die Validierung des Bilderwörterbuches zum Thema Nomen in der Schule besteht aus zwei Teilen. 
Zuerst ist die Evaluation der Wörter und der Sprache des Wortschatzes zum Thema Nomen in der Schule durchgeführt, was einemvon Muttersprachler gemacht wird. Danach wird das Design evaluiert, wass von einem Designexperten gemacht wird.

Der Muttersprachler, der die Daten schon korrigiert hat, gab folgende Kommentare: (1) das Video ist ein guter Lernmaterial, (2) es gibt noch wenige Schreibfehler und wenige Grammatikfehler, (3) der Wortschatz ist umffassend, enthält aber auch wenige unpassende Wörter. Der Experte hat dieCD des Bilderwörterbuches zum Thema „Nomen in der Schule” mithilfeder Applikation Videoshowschonkorrigiert, danach hat der Experte dieCD des Bilderwörterbuches zum Thema „Nomen in der Schule” benotet: Von 7 Kategorien der Aspekt der Evaluation gibt der Experte die Note 4 fürfünfAspekt, nämlichfür denInhalt des erstellten Bilderwörterbuches zum Thema Nomen in der Schule, den Wortschatz der Nomen in der Schule, das die Gelstaltung, die Leichtigkeit des Verständnisses und dieVollständigkeit des Wortschatzes.

Die Note 4 bedeutetsehr gut. Die Expertin gibt die Note 3 für die Bilder und dieVollständigkeit der Wortschatzes. Die Note 3 bedeutet gut. Die Note für das Bilderwörterbuches zum Thema Nomen in der Schule mithilfe Applikation Videoshow ist 92, 85 (sehr gut). Basierend auf der obigenMeinungkannfestgehaltenwerden, dass die CD zum Wortschatz zum Thema "Nomen in der Schule mithilfe der Applikation interessantgestaltetist. Die Note 4 bedeutetsehr gut. Der Expertegibt die Note 3 für die Klarheit des Wortschatzes und die Vollständigkeit des Wortschatzes.Basierend auf der obigenMeinungkannfestgehaltenwerden, dass die CD des Bilderwörterbuches zum Thema Nomen in der Schule mithilfe der ApplikationVideoshow interessantgestaltetist.

\section{Das Ergebnis der Erstellung}

Das Design des Lernmediums ist vielfältig und kann auf unterschiedliche Weise entworfen werden.

Das erste Video $=$ Der Wortschatz A und B. 
Das zweite Video = Der Wortschatz C, D, E, F.

Das dritte Video $=$ Der Wortschatz G und $\mathrm{H}$.

Das vierte Video 4 = Der Wortschtaz K.

Das fünfte Video 5 = Der Wortschatz L und M.

Das sechste Video 6 = Der Wortschatz N, O, P, R.

Das siebte Video 7 = Der Wortschatz $\mathrm{S}$

Das achte Video $=$ Der Wortschatz $\mathrm{T}$

Das neunte Video 9 = Der Wortschatz U, V, W,Z.

Das Bilderwörterbuch beginnt mit der Startseite. Die Startseite wird mit einem Satz, einem Bild und einem Symbol der Deustchlandflagge entworfen. Auf der Startseite wird auch der Name der Verfasserin geschrieben, dann folgen die Nomen in der Schule. Es gibt Singular und Plural, und endlich kommt der die Beispielsatz des Nomens in der Schule.

Im Video gibt es unterschiedliche Effek und unterschiedliche Musik, aber das Video hat die gleiche Schrift und die gleiche Farbe. Die Kapazität von allen Videos ist 221.64 MB. Jedes Video hat eine unterschiedliche Länge. Das erste Video dauert circa 05:24 Minuten, das zweite Video dauert 05:07 Minuten, das dritte Video dauert 02:50 Minuten, das vierte Video dauert 04:29 Minuten, das fünfte Video dauert 03:00 Minuten, das sechste Video dauert 02:36, das siebte Video dauert 04:41 Minuten, das neunte Video dauert 02:25 Minuten. Man kann alle Videos in “Galerie" anschauen, oder in der Applikation im Ornen “karya saya” öffnen.

In der Zukunft kann diese Untersuchung als Lernmedium besonders für Deutschlehrer und Deutschlernende benutzt werden. Durch diese Erstellung können die Schüler den Wortschatz zum Thema Nomen in der Schule leichter beherrschen.

\section{Die Diskussion}

Basierend auf den Ergebnisse der Erstellung eines Bilderwörterbuhes zum Thema Nomen in der Schule des Worschatzes in Form eines Videos mithilfe der Applikation Videoshow wurde zusammengefasst, dass die Daten in dieser 
Untersuchung die Wörter und Bilder über Nomen in der Schule sind. In dieser Untersuchung wird die Plomp Theorie benutzt.

Diese Theorie besteht aus vier Phasen, nämlich: (1) Die Beobachtungsphase, (2) die Designphase, (3) die Realisierungsphase- oder Konstruktionsphase und (4) die Revisionsphase. In diesem Kapitel wird jede Phase mit ihrem Ergebnis erklärt. In der Beobachtungphase werden einige Bücher gelesen, um Nomen in der Schule zu sammeln.

Nachdem werden die Bücher gelesen wurde, werden die Nomen im Buch markiert und gesammelt. Andere Nomen können aus anderen Quellen. Anschließen wird jedes Nomen vom Indonesischen übersetzt ins Deutsche.Nachdem die Daten reduziert wurden, werden130 Nomen gefunden undwirdAlphabetischgeordnet. Nicht alle Nomen können übersetzt werden.

In den zweiten Phase wird die Designphase durchgeführt. In dieser Phase werden die Bilder geordnet.. Das Designkonzept beginnt mit der Startseite, dann folgen die Nomen in der Schule. Es gibt Singular und Plural, und endlich kommt der Satz des Nomens in der Schule.Im Video gibt es unterschiedliche Effekt und unterschiedliche Musik, aber im Video hat gleiches Schreiben und gleiche Farbe. Das Design des Video ist schwierig. wenn ein Bild Falsch ist, also alle berarbeitung sind Falsch. z.B die Berabeitung die Dauer, Effekt, die Schreibung.Die Verwendung von "Multimusik" hat begrenztheit.

In den dritten Phase des Realisierungs- oder Konstruktionsphase wirddie Erstellung eines Bilderwörterbuches zum Thema Nomen in der Schule realisiert. Danach wird in der Revisionsphase schondas ein interessantes CD der Erstellung eines Bilderwörterbuches zum Thema Nomen in der Schule mit der Applikation Videoshowerstellt.

EswirdeineValidierungdurchgeführt. Der Experte hat das die interessanteLern- CD des Lernenmithilfe der Applikation Videoshow schonkorrigiert. Dann hat der Experte die interessante CD eines Bilderwörterbuches zum Thema "Nomen in der Schule" benotet: Von 7 Kategorien der Aspekte der Evaluation gibt der Experte die Note 4 fürfünfAspekt, nämlich der Inhaltdemerstellten Bilderwörterbuches zum Thema Nomen in der 
Schule, den Wortschatz des Nomens in der Schule mithilfe der Applikation Videoshow, das die Gelstaltung, die Leichtigkeit des Verständnisses und dieVollständigkeit des Wortschatzes. Die Note 4 bedeutetsehr gut.

Die Expertin gibt die Note 3 für die Bilder und dieVollständigkeit der Wortschatzes. Die Note 3 bedeutet gut. Die Note für eines Bilderwörterbuches zum Thema Nomen in der Schule mithilfe Applikation Videoshow ist 92, 85 (sehr gut). Basierend auf derobigenErklärungkannzusammengefasstwerden, dass die Erstellung eines Bilderwörterbuches zum Thema Nomen in der Schule in Form eines Videos mithilfe der ApplikationVideoshow mit der Plomps Theorie sehr gut ist, sodass die Lehrer/Lehrerinnen das Lernmedium benutzen können und die Schüler das Wortschatzzum Thema Nomen in der Schule leichter beherrschen können.

\section{SCHLUSSFOLGERUNG}

Nach den Untersuchungsergebnissen fallen die Schlussfolgerungen folgendermaßen aus:

1. Der Prozess der Erstellung eines Bilderwörterbuches zum Thema Nomen in der Schule mithilfe der Appllikation Videoshowbesteht aus der Erklärung des Phasen von Plomp. Darunter sind: (1) die Beobachtungsphase, (2) die Designphase, (3) die Realisierungsphase- oder Konstruktionsphase und (4) die Revisionsphase.

2. Das Ergebnisder Erstellung eines Bilderwörterbuches zum Thema Nomen mithilfe der Applikation Videoshowsind:

a. In dieser Untersuchungwurde eineinteressante $\mathrm{CD}$ des Bilderwörterbuches zum Thema "Nomen" mithilfe der Apllikation Videoshowerstellt, das in dieserAbschlussarbeitangehängtwird.

b. Dieinteressante CD des Wortschatzes zum Thema "Nomen" wurdevon einemPrüferkorrigiert, deshalbistdieseerstellte $\mathrm{CD}$ des des Bilderwörterbuches zum Thema "Nomen" mithilfe der Apllikation Videoshowsehr gut 
Studia: Journal des Deutschsprogramms

p-ISSN 2301-6108

e-ISSN 2654-9573

https://jurnal.unimed.ac.id/2012/index.php/studia/index

\section{LITERATURVERZEICHNES}

Choirul.N.A. 2014. Pengaruh Minat Baca dan Penguasaan Kosakata Terhadap Ketrampilan Menulis Bahasa Jerman peserta didik Kelas XI SMA NEGERI 2 Wonogiri. Skripsi : Universitas Negeri Yogyakata.

Fraudina,sara.dkk. 2015. Penggunaan Metode Word Square Bergambar dalam meningkatkan Penguasaan Nomina Bahasa Jerman.Skripsi: Universitas Pendidikan Indonesia.

Gunther. 1983. Duden Deutschches Universal Wörterbuch. Wien: Dudenverlag. Hüber. 1970. Deutsche Grammatik im Überblick. München.

Hutagalung, Surya. 2015. Metodologi Penelitian Dalam Praktek. Medan: Unimedpress.

Ismawarti, Dewi. 2014. Pengembangan Kamus Bergambar peralatan Membuat Dan Menyajikan Minuman Non-Alkohol Dan Minuman Beralkohol Untuk Siswa Jasa Boga Kelas Xi Smk Negeri 4 Yogyakarta. Skripsi: Universitas Negeri Yogyakarta.

Krisyanidayati.2017.Ketergantungan Tinggi Terhadap Gadget.Belitung.tribunnews.com/2017/03/08smp-di-tanjungpandan-miliki ketergantungan-tinggi-terhadap gadget wird am 10. April 2017 um 14.00 Uhr gelesen.

Musdeoranje.2017. AplikasiEditorVideoshow dan Langkah-Langkah edit Video hhtp : // www.musdeoranje.net/p/ccontact-me-html wird gelesen am 12.April 2017 um $11 \mathrm{Uhr}$ gelesen.

Rahayu sri. 2013.Penerapan Model Pembelajaran Kontekstual dalam Pembelajaran menulis pada Siswa kelas XII SMKN 1 Denpasar. ejurnal : (2) 2 .

Rochmad. 2012. Desain Model Pengembangan Perangkat Pembelajaran Matematika. Jurnal Krean, (1) 66-67. 
Studia: Journal des Deutschsprogramms

p-ISSN 2301-6108

e-ISSN 2654-9573

https://jurnal.unimed.ac.id/2012/index.php/studia/index

Singgih Yuntoto. 2015. Pengembangan Aplikasi Android sebagai Media Pembelajaran Kompetensi Pengoreperasian sistem pengendali elektronik pada siswa kelas XI SMKN 2 Penagsih. skripsi : Universitas Negeri Medan.

Sugiyono. 2016. Metode Pengembangan dan Penelitian. Bandung: Alfabeta.

Sukmadinata.N.S. 2009. Metode Penelitian Pendidikan. Bandung: PT Remaja Rosdakarya.

Tschmer, Erwin. 2010. Wortschatz. University of leipzig.

Vicosta efran. 2011. EYD dan Tata Bahasa Indonesia. Jakarta: Jal Publishing.

http://play.google.com/store/apps/details/id=com.xvideostudio.videoeditor wird am 10 April um 13.00 Uhr gelesen.

Gloria Agustina Saragih is an alumni of German language study program of Unimed, Medan. 